\title{
VOCACIÓN Y PRECARIEDAD LABORAL EN LA PROFESIÓN DE LA DANZA EN ESPAÑA: EFECTOS DE UNA POLÍTICA CULTURAL INEFICAZ
}

\author{
Patricia Bonnin-Arias \\ Universidad Rey Juan Carlos \\ Universidad Antonio de Nebrija \\ Juan Arturo Rubio-Arostegui
Universidad Antonio de Nebrija
}

\section{Resumen}

Uno de los ejes de la invención de la política cultural en el siglo XX consistió en el apoyo social y laboral al artista, desde un conjunto de acciones desde el lado de la oferta. En el caso español, y en concreto en el sector de la danza, en este trabajo se muestra la debilidad e ineficiencia por parte del Estado en dotar de unas condiciones laborales que permitan el desarrollo de una carrera artística al margen de la precariedad. Esta se evidencia tanto en las condiciones objetivas - debilidad de la oferta de compañías estables-e incremento insostenible de los conservatorios profesionales y superiores por parte de las Comunidades Autónomas, como en los propios discursos de los bailarines españoles en el paradigma del nuevo espíritu del capitalismo.

\section{Palabras clave: POLÍTICA CULTURAL; DANZA ESPAÑOLA; PRECARIE- DAD LABORAL; VOCACIÓN; BAILARINES}

\section{VOCATION AND PRECARIOUS LABOUR IN SPANISH DANCERS: OUTCOMES OF AN INEFFECTIVE CULTURAL POLICY}

\begin{abstract}
One of the drivers of the invention of cultural policy in the twentieth century was the social and labor support to the artist from a set of actions from the supply side. In the Spanish case, and specifically in the dance sector, this article shows the weakness and inefficiency on the part of the State in providing working conditions that allow the development of an artistic career in the this artistic field without precariousness. It is evident both in the objective conditions -weak supply of stable companies- and unsustainable increase in professional and superior conservatories by the Autonomous Communities, as in the discourses of the Spanish dancers in the paradigm of the new spirit of capitalism.
\end{abstract}

Keywords: CULTURAL POLICY; SPANISH DANCE; PRECARIUS LABOUR; VOCATION; DANCERS

\footnotetext{
Bonnin-Arias, Patricia; Rubio-Arostegui, Juan Arturo. 2019. "Vocación y precariedad laboral en la profesión de la danza en España“. AusArt 7 (2): 103-112. DOI: 10.1387/ausart.21132
}

\section{AUSART}


Las profesiones de la danza se encuentran en España en vías de dignificación, tras haberse desarrollado en un contexto social, cultural y político que ha tenido como principales características la precariedad, la inestabilidad, la dependencia y la invisibilidad (Plan General de la Danza 2010-2014 [INAEM 2009]). Pese a que las políticas culturales europeas se han apoyado en la oferta (Menger [2001] 2016) y en intentar proveer un entorno propicio para la mercantilización de la cultura en condiciones de fallo del mercado en el caso de las artes escénicas (Baumol \& Bowen 1966), esta política presenta síntomas de agotamiento, insostenibilidad, sobre todo a partir de la crisis de la financiación de la cultura (Rubio-Arostegui, Rius-Ulldemolins \& Martínez-Illa 2014).

En el caso de España, la acción cultural en torno a la danza escénica tras el paréntesis franquista significó una oportunidad para un arte cuyo apoyo institucional durante la dictadura no fue más allá de puntuales acciones, principalmente en torno a la danza tradicional y española (Santa-Cruz 1995). En la democracia, la implementación en los años 80 de políticas de fomento a la danza a nivel estatal, autonómico y local, contemplando -entre otras medidas- la creación y sostenimiento de los elencos nacionales de danza española y ballet, los programas de ayuda a las compañías privadas de danza de diverso formato, el desarrollo de centros de creación y plataformas de apoyo a la danza en algunas comunidades autónomas y los programas de residencia en teatros de titularidad municipal, esbozó un panorama institucional público-privado distinto (INAEM 2009).

Aun reconociendo tímidas mejoras globales, parecen persistir las carencias estructurales en un entorno hostil para la danza, tras la crisis global en la que se constata casi una paralización del mercado de las artes escénicas, del que la danza sale aun peor parada. Así, una de las redes de teatros pioneras en España como es la de la Comunidad de Madrid ha pasado de un gasto de 4.896.239 € en 2010 a una caída del 67,32\% en el año 2017. Asimismo, en los propios municipios de la región se ha pasado de un gasto de $84.418 €$ en 2010 a $24.343 €$ en 2017, lo que supone una reducción del 71,16 (Moraga Guerrero 2018).

La creación del Ballet Nacional de España y de la Compañía Nacional de Danza, ambas de titularidad estatal a fines de la década de los 70 , no ha podido servir como respuesta a la situación de precariedad de los bailarines, sobre todo si lo comparamos con el caso francés donde en los años 80 se desarrolló una política de desconcentración a través de los Centros Coreo- 
gráficos Nacionales como consecuencia de los acuerdos entre el Ministerio de Cultura y las entidades territoriales. Por otro lado, las compañías privadas tampoco pueden garantizar unas condiciones laborales dignas, sujetas al arbitrio de la convocatoria de ayudas y de otras decisiones políticas. En la matriz generada por este panorama institucional público-privado, que dibuja una tortuosa trayectoria de la política cultural española, se introduce la problemática de las condiciones laborales del personal que lo integra, cuestión que nos ocupa en este artículo.

En el mismo se ofrece un panorama de la incidencia de las políticas culturales y educativas en la articulación de la relación oferta-demanda de la danza en España, además de los resultados de un estudio basado en el análisis sociológico del discurso de veintidós agentes consagrados del ámbito de la danza clásica en España respecto a las condiciones laborales de su profesión.

\section{EL ESPACIO ESTRUCTURAL DE LA DANZA EN ESPAÑA: LA RELACIÓN DE LAS POLÍTICAS PÚBLICAS EN LA RELACIÓN ENTRE LA OFERTA Y LA DEMANDA}

La precariedad se manifiesta como fruto de las condiciones objetivas de la mercantilización del arte, traducida en la relación entre oferta y demanda, siendo la primera mucho más incentivada por las políticas públicas. Esto se evidencia en que la danza ha sido abordada con mayor énfasis por la política educativa, a través de las enseñanzas artísticas profesionales y superiores. La oferta de conservatorios de danza ha tenido un cierto despliegue con el protagonismo de los gobiernos autonómicos que han destinado una parte importante de los recursos del sector en la formación reglada (Rubio-Arostegui 2016). Ello trae como consecuencia directa un incremento en los profesionales dispuestos a comenzar una carrera profesional en el mundo de la danza y un alza en la tasa de paro del sector como consecuencia de un mercado que no termina de funcionar desde las redes de teatros de titularidad pública. Así, se constata un incremento de $41,3 \%$ de alumnos matriculados en enseñanzas regladas entre 2002 y 2014 en el global nacional, con números más acusados en Valencia y Andalucía (Rubio-Arostegui 2016). Por su parte, el sobredimensionamiento de la formación profesional en danza da lugar a un exceso de mano de obra, 
imposible de absorber por el mercado laboral, hecho que se constata en los exiguos datos de afiliación de este colectivo (Rubio-Arostegui 2015).

Una evaluación de las enseñanzas artísticas, por tanto, de la propia política educativa descontextualizada de sus efectos en el mercado laboral, es de urgente necesidad y debería ser acometida por los ministerios e instituciones culturales y educativas de los gobiernos autonómicos. A ello hay que añadir la ausencia de una política cultural de fomento de la demanda como consecuencia de una gestión poco profesional de las redes y los teatros de titularidad pública (Rius Ulldemolins y Rubio-Arostegui 2016).

Otro espacio de generación de audiencias es el de la educación no formal, que en la danza lo ocupan las academias privadas, así como las escuelas de música y danza reguladas desde los 90 por la LOGSE. Pese que en ambos tipos de centro existe gran autonomía de currículo, la tendencia generalizada suele ser la de emular las prácticas de la educación formal de danza -los conservatorios- obviando la orientación democratizadora que deberían tener estos centros.

Tampoco encontramos un buen diagnóstico de las escuelas de música y danza públicas. Un indicador fidedigno de calibrar las dimensiones de la educación artística no formal, lo encontramos en el ámbito musical. En este sentido y en comparación con el modelo liberal, tanto escandinavo como central de política cultural, España adolece de una pobre densidad institucional en escuelas de música, situación extrapolable a la de la danza. Asimismo, pese a las deficiencias en la comparación de los datos por países de la Asociación de Escuelas de Música Europeas (EMU- European Music School Union 2010) las evidencias permiten establecer diferencias institucionales España y el resto de países del norte europeo.

\section{UNA PROFESIÓN ARTÍSTICA ESPECIALMENTE PRECARIZADA}

Todo el análisis de las condiciones objetivas y estructurales de la profesión de los bailarines se correlaciona con actitudes fuertemente vocacionales que, con el argumento de labors of love originan un compromiso mucho mayor con su arte que con las retribuciones económicas (Freidson 1990). 
La precocidad, la volatilidad del empleo, la dificultad en la promoción profesional y la brevedad de la vida útil del bailarín profesional del ballet académico no son objeto de compensación, ni a nivel salarial -comparativamente con grupos ocupacionales que requieren niveles similares de formación profesional y cualificación-, ni en cuanto a las condiciones de retiro (Menger 1999). De este modo, los bailarines dedicados al ballet académico obtienen bajos rendimientos de sus inversiones en educación en relación a otras profesiones comparables incluso entre las propias profesiones artísticas (Throsby 1994).

Se trata de un oficio que incluye recompensas simbólicas y materiales inciertas, que atraen a un gran número de aspirantes que logran sobrevivir a través de fórmulas que no son incompatibles entre sí y pueden ser combinadas, entre las que se incluyen el apoyo de familiares o amigos, subvenciones públicas, patrocinio de fundaciones o corporaciones privadas, seguros sociales, prestaciones por desempleo, además de empleos secundarios (Alper et al. 1996) como recursos de supervivencia durante la espera del reconocimiento de su talento, ya que, en el mercado del arte, el ganador se lo llevará todo. Así pues, toda la inventiva se basa en el desarrollo de fórmulas contractuales y relacionales para gestionar esa incertidumbre extrema previa al reconocimiento (Menger 2009).

Paralelamente al desempleo y sus inquietantes datos en cuanto a prestaciones y dependencia económica, el informe de la Fundación AISGE (2016) pone de manifiesto la precariedad del empleo debido al endurecimiento de las condiciones laborales de los actores y bailarines en España con respecto a 2005. En el estudio se afirma que un $73 \%$ del colectivo de intérpretes no puede vivir de su trabajo.

\section{ANÁLISIS CUALITATIVO DE LA CONSTRUCCIÓN SOCIAL}

Abordando ya la dimensión subjetiva de esta problemática, encontramos dos factores definitorios de las profesiones artísticas: el componente vocacional -la illusio de Bourdieu- y la fascinación por el glamour y la propia experiencia estética que produce el arte, que explican el compromiso personal desinteresado, en el que el salario se percibe como algo necesario pero secundario (Rubio-Arostegui 2015). En España los bailarines parecen dispuestos a asumir 
las precarias condiciones laborales que les impone el mercado, sobre todo si esto implica un vínculo con una compañía de envergadura -como puede ser una compañía nacional-, mostrándose menos dispuestos en elencos privados con menor capacidad retributiva en términos de capital simbólico.

(...) yo he bailado sin cobrar ni un duro y era la mujer más feliz del mundo. $Y$ hay cantidad de bailarines aquí ahora mismo que bailan sin cobrar. (...) aunque empezasen pagándoles poco, el bailarín bailaría, seguro.

(BN-MSTR4)

Ellos [los bailarines de la Compañía Nacional de Danza] son la élite de su arte, de su profesión en el país, y no cobran como élite. Tienen unos sueldos, yo creo que en el cuerpo de baile son mileuristas.

(TRSGTS2)

La gente pues, "bueno, si no puedo vivir de la danza, lo voy a hacer gratis". (...) Y mucha gente joven que "bueno, esto te sirve de experiencia y baila conmigo, no te pago, pero te sirve de experiencia" ¿Pero hasta dónde? En cambio, viene un burro como nosotros y decimos: "no, aunque seas joven, tú tienes que estar contratado para salir a un escenario"; eso nos ha llevado a un declive económico brutal (...).

(DRTR-CRGF1)

Por otra parte, el estatus de nacional de una compañía de ballet ha colaborado con una relativa dignificación de la profesión. Si bien la situación aún sigue siendo precaria, la creación de los elencos nacionales y los movimientos asociativos en torno a ellos, han servido para visibilizar la profesión del bailarín, regular sus condiciones laborales a través de convenios y su cotización a la Seguridad Social.

(...) no existimos... no estamos en la Seguridad Social, ni hemos estado en la vida. No sé cómo nosotros vamos a cotizar, porque Bailarín no existe. Entonces en (...) los años 60, 70 y 80 (...) cotizábamos como Circo y Variedades, o sea que éramos "circanos" [sic]. (...). Pero hombre... éramos bailarines clásicos.

(BN-MSTR4)

Y lamentablemente hoy muchos de aquellos bailarines que bailaron entonces [durante la dictadura franquista], no tienen jubilación porque los que los contrataban no pagaban la Seguridad Social por 
ellos. (...) Entonces, gracias a los campos de ballets nacionales eso, los bailarines adquieren unos derechos. Hay unos sindicatos, hay unos convenios laborales.

(BN-MSTR6)

Pese a ello, los convenios colectivos que regulan la actividad de los artistas, no siempre se encuentran adaptados a las necesidades de trabajo del bailarín $y$, como ejemplo, el caso de las horas extra de los elencos nacionales compensadas con periodos de descanso, incompatibles para las necesidades de entrenamiento cotidiano del bailarín. Respecto al retiro, además de los requerimientos físicos extremos de su trabajo y de la caducidad de las competencias corporales, debe tenerse en cuenta que la longevidad de la carrera de un bailarín varía de acuerdo con el subgénero de danza al que se dedica, teniendo los bailarines de danza contemporánea una carrera más prolongada que la de los dedicados al ballet académico (Menger 1999). El actual régimen general de la Seguridad Social prevé la jubilación anticipada para los bailarines a partir de los sesenta años de edad ${ }^{1}$ todavía muy alejados de los cuarenta y dos años reglamentarios para jubilarse en la Ópera de París.

42 años. Lo tienen también clarísimo en (...) todos los lugares institucionalizados. Es decir, el cuerpo de un bailarín, para estar en el máximo, llegas a una edad en la que ya no puede.

(TRSGTS3)

Es un mundo es muy cruel, pero pienso que sí debería haber que se pudieran retirar después de un tiempo. (...) De los 45 años (...). Para el bailarín 45 años puede ser los 65 de los demás.

(TRSGTS2)

(...) un concertino cobra $3.000 €(\ldots)$ que es equivalente a un primer bailarín (...) ¿Cómo un primer bailarín va cobrar 1.600? Es vergonzoso cuando un músico puede estar tocando hasta los 80 años y un bailarín a los $40 / 42$ ya se tiene que retirar (...) Un bailarín no tiene sentido que se jubile (...) a los 65 . (...) porque un bailarín, cuando empezamos a trabajar con 16-18 años, cuando otros estudiantes hasta que no terminan la carrera con 26-28 años... nosotros llevamos cotizando ya 10 años antes que el resto.

(BN-MSTR6)

Una de las reivindicaciones más frecuentes de los integrantes de elencos dependientes de las administraciones públicas trata de la funcionarización de la plantilla, como vía de compensación a la inestabilidad laboral. Si bien 
décadas atrás se produjeron nombramientos tanto en los elencos nacionales como en el Ballet de Zaragoza, el choque entre la lógica administrativa y artística ha derivado en disfuncionalidades de las relaciones contractuales, llevando frecuentemente ante la Justicia a las administraciones y a los artistas, al tiempo de generar dificultades en la reubicación de artistas funcionarios en otros puestos de la Administración. Desde 1995 y hasta la actualidad, la contratación de los bailarines de las compañías nacionales es de naturaleza temporal y su renovación cíclica se realiza en fraude de ley por parte de la Administración General del Estado, incapaz de reconocer la naturaleza indefinida de contratos temporales que han llegado a renovarse anualmente hasta catorce años seguidos. En 2016, las reivindicaciones de los artistas adscritos a los elencos nacionales, han suavizado el argumento del derecho a la funcionarización, inclinándose por el reconocimiento de la naturaleza indefinida de estos contratos.

Al finalizar su carrera de bailarín, aquellos profesionales que habían conseguido un puesto en la Administración, han debido enfrentarse a la reubicación en un nuevo puesto dentro de ella, para su "reciclaje". Estos procesos se han dado de forma heterogénea, o bien integrándolos en puestos administrativos, o bien en relaciones más fructíferas la colaboración de los profesionales con los conservatorios públicos de danza

(...) lo que se pensaba en principio era formarles para que pudieran asumir otros papeles dentro del mundo de la danza, estaba muy bien pensado, pero luego la realidad es que los colocaron en puestos administrativos. Entonces, hubo bailarines que acabaron con una depresión impresionante (...). (...) un bailarín que está acostumbrado a giras, bailar todo el día, de pronto le colocas delante de una fotocopiadora sólo para hacer fotocopias y es algo que es un maltrato (...). Afortunadamente se han podido recuperar bailarines que ahora están en producción o bailarines que han pasado a conservatorios.

(TRSGTS3)

Entonces a algunos que estaban en la Administración se les pedía que colaboraran en los conservatorios en la parte artística, en la parte de los talleres para hacer las coreografías, para hacer repetición de ballet (...). Durante un tiempo, con buena armonía de los que estaban arriba se hizo un convenio. (...). Entonces así fue 
como la figura del "reciclado" se incorporó en los conservatorios y dio gran ayuda (...).

(DRTCNSR1)

Queda por ver que la reciente aprobación del Estatuto del Artista abra un nuevo capítulo para la estabilidad y dignificación laboral del bailarín, si las condiciones objetivas del mercado nacional no cambian. La valorización de la profesión del bailarín no parece ser la misma en España que en aquellos países con una mayor protección hacia sus derechos fundamentales.

\section{CONCLUSIONES}

Existe una amplia literatura de las ciencias sociales desde primeros de siglo (Ellmeier 2003) que evidencia que los artistas en el régimen de trabajo posfordista se han convertido en individuos emprendedores que trabajan bajo condiciones laborales precarias en trabajos por proyectos en el marco de una vocación artística que funciona como un a priori. En este artículo se aportan datos empíricos sobre la situación española del bailarín, que corroboran la precariedad en el marco del paradigma del nuevo espíritu del capitalismo (Boltanski \& Chiapello 2002).

\section{Referencias bibliográfícas}

Alper, Neil O. et al. 1996. Artists in the work force: Employment and earnings 1970-1990. Research Division Report (National Endowment for the Arts) 37. Santa Ana CA: Seven Locks. www.arts.gov/sites/default/files/NEA-Research-Report-37.pdf

Baumol, William J. \& William G. Bowen. 1966. Performing arts: The economic dilema; A study of problems common to theater, opera, music and dance. Cambridge MA: MIT

Boltanski, Luc \& Eve Chiapello. 2002. El nuevo espíritu del capitalismo. Traducción Marisa Pérez Colina, Alberto Riesco Sanz, Raúl Sánchez Cedillo; edición de Carlos Prieto del Campo. Madrid: Akal

Ellmeier, Andrea. 2003 "Cultural entrepreneurialism: On the changing relationship between the arts, culture and employment". International Journal of Cultural Policy 9(1):3-16, DOI:10.108 0/1028663032000069158a

EMU (European Music School Union). 2015. "Statistical information about the European Music School Union”. Texts, Helena Maffli. www.musicschoolunion.eu/wp-content/ uploads/2017/09/EMU-Statistics-2015-08.09.2017.pdf

Freidson Eliot. 1990. "Labors of love: a prospectus". En The Nature of work: Sociological perspectives, Kai Erikson \& Steven Peter Vallas, 149-61. New Haven CT: Yale University 
Fundación AISGE (Artistas Intérpretes, Sociedad de Gestión). 2016. "Estudio y diagnóstico de la situación sociolaboral de actores y bailarines en España”. Aisge.es, 29 sept. www.aisge. es/informe-sociolaboral-aisge-2016

INAEM (Instituto Nacional de las Artes Escénicas y de la Música). 2009. "Plan General de la Danza 2010-2014". Aprobado en el Foro Estatal de la Danza (Tenerife, 2-3 nov). www.cioff. es/documentos/descargas/6-Plan\%20Danza\%20Inaem.pdf

Menger, Pierre-Michel. (2001) 2016. “Art, politització i acció pública”. Traduït del francés per Cristina Guirado Gil amb la col·laboració de Maria Josep Cuenca. Debats 130(2): 73-98

- . 1999. "Artistic labor markets and careers". Annual Review of Sociology 25: 541-74. www. jstor.org/stable/223516

— . 2009. Le travail créateur: S’accomplir dans l'incertain. París: Gallimard

Moraga Guerrero, Eva, dir. 2018. "Propuestas para la mejora de la Red de Teatros de la Comunidad de Madrid y de la situación de la danza en la Red”. Asociación Emprendo Danza. http://emprendodanza.feced.org/wp-content/uploads/sites/8/2018/10/Informe_Red_Teatros_Eva_Moraga_EmprendoDanza.pdf

Rius Ulldemolins, Joaquim \& Juan Arturo Rubio-Arostegui, eds. 2016. Treinta años de políticas culturales en España: Participación cultural, gobernanza territorial e industrias culturales. València: Universitat de València

Rubio-Arostegui, Juan Arturo, Joaquim Rius Ulldemolins \& Santi Martínez Illa. 2014. El modelo español de financiación de las artes y la cultura en el contexto europeo: Crisis económica, cambio institucional, gobernanza y valor público de la cultura y la política cultural. Con la colaboración de $\mathrm{M}^{\mathrm{a}}$ Victoria Sánchez Belando. Madrid: Fundación Alternativas. www.fundacionalternativas.org/public/storage/cultura_documentos_archivos/3c1678b9d12200244b199cde7bbbc27a.pdf

Rubio-Arostegui, Juan Arturo. 2015. "Informe socio-económico sobre las profesiones artísticas en el contexto de la crisis sistémica de la cultura en España". Inédito

— . 2016. "Dimensiones y papel de la educación artística en las políticas educativas y culturales en la España democrática”. En Treinta años de políticas culturales en España, Joaquim Rius Ulldemolins \& Juan Arturo Rubio-Arostegui, eds. ,321-39. València: Universitat de Valéncia.

Santa-Cruz, Lola. 1995. "Festivales de España: Una mancha de color en la España gris". En Historia de los Teatros Nacionales: Volumen segundo; 1960-1985, ed, de Andrés Peláez, 189-208. Madrid: Centro de Documentación Teatral

Throsby, David. 1994. "The production and consumption of the arts". Journal of Economic Literature 32(1), 1-29

\section{Notas}

1 “Jubilación anticipada por razón del grupo o actividad profesional". Web Seguridad Social. http://www.seg-social.es/wps/portal/wss/internet/Trabajadores/PrestacionesPensionesTrabajadores/10963/28393/28464

(Artículo recibido: 08-10-19; aceptado: 19-11-19) 